REVISTA DE LITERATURA E CULTURA RUSSA

\title{
La contradiction et la limite Sur la démonologie poétique de M. Y. Lermontov
}

\section{The contradiction and the limit On the poetic demonology of $\mathrm{M}$. Y. Lermontov}




\section{La contradiction et la limite sur la démonologie poétique de $M$. Y. Lermontov}

lacopo Costa*

Résumé: L'article entend démontrer que la poésie de Lermontov propose une philosophie du démoniaque. L'enjeu du démoniaque apparaît particulièrement adapté à une approche de l'œuvre de Lermontov, non seulement en raison de la présence constante du diable ou du monde démoniaque dans la pensée occidentale , mais aussi en raison de sa centralité évidente dans l'œuvre de Lermontov. II s'agira alors de déceler les traces de cette réflexion et de ses interrogations fondamentales dans la fiction, ainsi que dans le lexique et les images dont Lermontov se sert.

\begin{abstract}
Poet and novelist Mikhail Yuryevich Lermontov (1814-1841) is recognized as one of the most important authors of Russian Romantic literature. The figure of the devil occupies a central place in his production. From a number of poetic texts (My Demon, The Angel of Death, The Demon ...), we propose to outline the basic features of a philosophy of the demonic: in Lermontov, the voice of the demon is a means to express the radical separation of man from nature. The contradiction, that is to say the negation of rationality, appears to be one of the fundamental elements by which this separation is expressed.
\end{abstract}

Mots-clefs: Lermontov; Poésie et philosophie; Démonologie; Contradiction Keywords: Lermontov; Poetry and philosophy; Demonology; Contradiction; 
* Centre National de la Recherche Scientifique - CNRS / Laboratoire d'Études sur les Monothéismes LEM (UMR 8584). E.mail: iacopo. costa@gmail.com

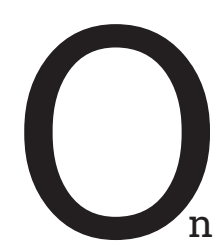

sait tout, ou presque, de la brève vie de Mikhaïl Yourievitch Lermontov. Outre un certain nombre de grands poèmes lyriques (L'ange, Les trois palmiers, Le rêve ...), il est principalement connu pour deux œuvres: un grand poème, Le Démon, dont la huitième et dernière version, en 1132 vers, date de 1839; et un roman, Un héros de notre temps, publié en épisodes en 1839, puis en volume en 1840 . On dit qu'il aurait clôt, par Le Démon, lâge du roman en vers porté à sa perfection par Pouchkine, et ouvert, par Un héros de notre temps, celui du grand roman psychologique. ${ }^{1}$

Je soutiens ici que la poésie de Lermontov propose une philosophie du démoniaque. Deux prémisses semblent s'imposer. Premièrement, Lermontov a eu une vie brève, qu'il a consacrée en partie à sa carrière militaire, en partie aux mondanités que son rang et sa position lui imposaient, en partie, et surtout, à la composition de son œuvre littéraire et poétique. Il ne semble pas avoir accompli d'études philosophiques approfondies, mais il n'est pas interdit de penser que sa vivacité intellectuelle extrême, ainsi que sa fréquentation des élites russes, l'ont porté à absorber, d'une manière ou d'une autre, une partie de la philosophie de son temps. Deuxièmement, nous croyons, malgré cela, que la puissance de sa parole est telle qu'à sa racine se trouvent des interrogations radicales et puissantes sur son être de poète et sa relation au monde. C'est une partie de ces interrogations qu'on souhaite ici éclairer.

L'enjeu du démoniaque apparaît particulièrement adapté à une approche de l'œuvre de Lermontov, non seulement en raison de la présence constante du diable ou du monde démo-

1 Le lecteur francophone dispose de deux éditions particulièrement remarquables: pour la poésie, LERMONTOV, 1985 (dorénavant (EP); pour la prose, GRIBOÏĖDOV, POUCHKINE, LERMONTOV, 1973. Voir aussi LERMONTOV, 2003 (édition bilingue, enrichie d'appareils critiques). Sauf indication contraire, les traductions des textes de Lermontov sont de moi. Je me réfère au texte imprimé dans LERMONTOV, 1954-1957 (dorénavant Sotch.). 
niaque dans la pensée occidentale, ${ }^{2}$ mais aussi en raison de sa centralité évidente dans l'œuvre de Lermontov. Dans son livre Russian Literature, P. Kropotkine parle très à propos du 'démonisme' et du 'pessimisme' de Lermontov, ${ }^{3}$ même si l'anarchiste qu'était Kropotkine est surtout intéressé par le démonisme comme origine supposée de la forte tendance du poète à la rébellion sociale. Bien que le démonisme lermontovien reflète sans doute une attitude politique et existentielle séditieuse, ${ }^{4}$ il est impossible de le réduire à cela: son démonisme dérive aussi de sa réflexion sur la vie humaine et sa relation à la nature. Malgré cette orientation radicale, la poésie de Lermontov n'est jamais 'doctrinale'. Il s'agira alors de déceler les traces de cette réflexion et de ses interrogations fondamentales dans la fiction, ainsi que dans le lexique et les images dont Lermontov se sert.

Lermontov est le poète de l'étrangeté désespérante de l'homme et de la nature: le monde de l'homme et le monde de la nature sont séparés, l'un déploie son existence dans l'autre, mais ils ne pourront jamais coexister harmonieusement. C'est l'idée à laquelle fait allusion, avec une déconcertante simplicité, le bref poème Le ciel et les étoiles (1831):

Pur est le ciel du soir,

Claires les étoiles lointaines,

Claires comme le bonheur d'un enfant;

Oh! pourquoi ne puis-je pas penser:

Étoiles, vous êtes claires, comme mon bonheur!

Pourquoi es-tu malheureux,

Me diront les gens?

Pour cela je suis malheureux,

Mes braves gens, que les étoiles et le ciel

Sont les étoiles et le ciel! et moi, je suis un homme! ...

2 Voir l'ouvrage récent, bref mais profond, de GREGORY, 2013.

3 KROPOTKIN, 1905, p. 55: «Lérmontoff's demonism or pessimism was not the pessimism of despair, but a militant protest against all that is ignoble in life, and in this respect his poetry has deeply impressed itself upon all our subsequent literature».

4 Sa biographie fournit abondance d'exemples de ces tendances. N'oublions pas que le véritable début poétique de Lermontov correspond à la diffusion de son poème La mort du poète (1837), écrit à l'occasion de la mort de Pouchkine, contenant une invective violente contre la cour de Nicolas ler, qui fut à l'origine de son premier exil dans le Caucase. 
Les gens l'un envers l'autre

Nourrissent l'envie;

Et moi, au contraire,

Je n'envie que les belles étoiles,

Ce n'est qu'à leur place que j'aurais voulu être. ${ }^{5}$

On retrouve ici à la fois le sentiment de supériorité que Lermontov ressentait vis-à-vis de ses semblables qui, s'enviant mutuellement, illustrent l'illusion d'une satisfaction qu'apporteraient les biens humains, et la radicalité de son désespoir devant la nature, puisque son seul désir - être parmi les étoiles - est un désir impossible. Dans cette relation de négation réciproque, l'être démoniaque intervient avec un but précis: il assure à la fois la scission consciente de l'homme et de la nature et la possibilité de voir et d'exprimer la nature. Il garantit ainsi l'accès de l'homme à la nature, mais avec le seul objectif de lui interdire la perfection de la nature.

Le démon n'est pourtant pas un simple antagoniste de Lermontov: au contraire, il instaure avec le poète un jeu cruel d'identification et de distinction, une sorte de possession par l'image et par la parole dans laquelle s'accomplit la destinée à la fois poétique et existentielle du poète. Tentons alors de saisir, sinon de définir, la dimension démoniaque lermontovienne. La tâche est rendue moins ardue par Lermontov luimême.

\section{Le Démon, le Poète et la Muse}

L'un des premiers 'moments démoniaques' dans la pensée de Lermontov est constitué par les deux rédactions d'un bref poème, Mon démon (1829, 1830-1831). Nous sommes autorisés à voir dans ce poème, sinon un programme idéal et littéraire, du moins la révélation de l'un des ressorts les plus profonds de l'inspiration du poète. On notera aussi une évolution importante entre la première et la seconde rédaction, puisque dans la seconde le démon prend les traits du protagoniste de l'Ange

5 Sotch., t. I, p. 219 
de la mort, poème sur lequel nous viendrons par la suite, au point de se confondre avec celui-ci. Mais Lermontov semble ici nous suggérer aussi que cette créature purement négative l'inspire et le possède, au point que lui-même se sent envahi par son être.

La première rédaction fournit une caractérisation physique (v. 1-8) puis intellectuelle (9-16) du démon de Lermontov:

Son élément est un concentré de maux.

Courant à travers les nuages fumeux,

Il aime les ouragans meurtriers

L'écume des rivières et le bruissement des chênaies.

Dans un tourbillon de feuilles jaunes,

Se dresse, immobile, son trône;

Au milieu de vents sans voix,

Il siège, funeste et sombre.

Il instille la méfiance,

Il méprise l'amour pur,

Il réprouve les prières,

Il reste insensible à la vue du sang,

Et le chant des nobles sentiments,

Il le suffoque par la voix des passions,

Et la muse des douces inspirations

Redoute ses yeux inhumains.

Le premier vers («Собранье зол его стихия»), dont nous avons délibérément donné une traduction peu poétique, mérite d'être compris dans son sens littéral ${ }^{6}$. En effet, la suite du poème, qui expose les maux dont l'assemblage, ou la réunion, constitue l'être du démon, est un développement de ce premier vers. La stabilité ou pureté élémentaire du démon se fige dans l'image du trône, dont l'immobilité est accentuée par l'instabilité de la nature environnante (v. 5-6). Cependant, le démon est aussi saisi dans sa course, c'est-à-dire dans sa mobilité. Cette association du lourd et du léger, du mobile et de l'immobile, cette coïncidence de caractères contradictoires, concourt à un effort paradoxal, celui de penser la réalité démoniaque dans les termes mêmes de son inconcevabilité. Du reste, le concept

6 Voir $\mathbb{E} P$, p. 128: «Son élément est la misère» (tr. J.-Cl. Lanne); «Son seul élément est le mal» (tr. M. Cadot). 
même de 'concentré' ou 'réunion de maux' est auto-contradictoire, puisqu'il s'agit de tirer une substance à partir d'éléments qui ne subsistent pas.

Du point de vue intellectuel, le démon se présente comme un destructeur du bien et des valeurs (v. 9-11): il inspire aux hommes la méfiance réciproque, brisant les lieux affectifs et sociaux; il méprise l'amour pur; et il est insensible aux prières. Dans ces huit premiers vers, Lermontov met en place le triangle constitué par les trois réalités antagonistes que sont la nature, la vie humaine et le démoniaque: ces trois pôles qui entrent en tension les uns avec les autres tirent leur dynamisme de cette même tension.

L'action du démon a déjà commencé à affecter le poète qu'est Lermontov: les deux derniers vers montrent une muse apeurée face aux yeux d'outre-tombe du démon. ${ }^{7}$ Dans cette première rédaction du poème, le démon est encore un rival de la muse traditionnelle.

Dans la rédaction de 1829, Lermontov ne nous dit rien de sa propre relation au démon: seul le titre (Mon Démon) nous dit que c'est le démon de Lermontov. La seconde version éclairera cette relation, et en même temps nous donnera des éléments pour établir un lien entre ce démon de Lermontov et l'Ange de la mort.

La nouvelle rédaction, légèrement plus longue, conserve une partie seulement des vers de la rédaction originaire:

1

Son élément est un concentré de maux;

Courant à travers les sombres nuages,

Il aime les ouragans meurtriers

L'écume des rivières et les chênaies bruissant;

Il aime les nuits obscures,

Les brouillards, la lune pâle,

Les sourires amers et les yeux

Qui ne connaissent ni larmes, ni rêve.

7 Je rends ainsi l'adj. 'неземной', littéralement 'non terrestre', qui peut renvoyer tant à des êtres célestes et angéliques qu'à des êtres de l'au-delà. 
2

Aux arides bavardages du monde Il a pris l'habitude de prêter l'oreille, Il trouve ridicules les mots de salut Et tout croyant ridicule; Étranger à l'amour et au regret, Il vit de nourriture terrestre, Avale avidement la fumée de la bataille Et la vapeur du sang versé.

3

Si naît un nouveau martyr, Il va inquiéter l'esprit du père, Âprement le raillant, Avec un air de gravité barbare; Et lorsqu'une âme tremblante S'apprête à descendre dans la tombe, Il passe avec elle la dernière heure, Mais sans apporter au mourant du réconfort.

4

Et tant que je serai en vie,

Il ne me quittera pas, l'orgueilleux démon, Et mon intellect, il l'éclairera

Par le rayon d'une flamme prodigieuse;

L'image de la perfection, il me la montrera

Et soudain à jamais me l'ôtera

Et, m'ayant donné un avant-goût du bonheur,

Jamais ne me donnera la félicité. ${ }^{8}$

Lisons la quatrième strophe, où est institué un lien entre le démon et l'activité poétique de Lermontov. La muse de la première rédaction a disparu, et pour cause: elle a été remplacée par la nouvelle muse démoniaque; Lermontov semble avoir découvert que sa vraie muse est son démon. De sa relation au démon, Lermontov nous dévoile trois aspects. Premièrement, le démon-muse l'accompagnera jusqu'à la mort: c'est donc un destin, à la fois individuel et poétique. Deuxièmement, il éclaire l'intellect du poète, ou son esprit (ум): ce destin est un privilège, puisque la flamme de l'inspiration est prodigieuse (чудесный). Troisièmement, le démon-muse montre des

8 Sotch., t. I, p. 318-319. EP, p. 182-183. 
images, images de perfection, qui sont donc les visions poétiques de Lermontov. Or ces images de perfection sont à la fois aperçues, saisies et à jamais perdues: qu'est-ce que cela veut dire? Il faut probablement envisager une distinction entre un premier stade poétique, qui consiste en l'appropriation exclusivement poétique de l'image de perfection, et un second stade existentiel, où la perfection est perdue. Lermontov se sait conscient de parvenir, par ses vers, à exprimer la perfection, et se sent destiné à devoir abdiquer, comme homme, cette même perfection. Le démon est à la fois condition de perfection de son inspiration et condition de son malheur personnel; nous découvrirons que, dans cette configuration poétique et psychique, la perfection est constituée par la nature et le malheur par l'expérience humaine. Selon Lermontov, la beauté de la nature est inaccessible à l'homme autrement que par la poésie; la poésie consiste à savoir voir la beauté, mais cette vision implique la prise de conscience de l'impossibilité humaine de participer à la beauté et à la perfection de la nature.

Il ne faut pas oublier le point fondamental: la perfection vient de la flamme du démon. Cela signifie que le démoniaque, loin de représenter une réalité en soi exclusivement destructrice, représente au contraire une réalité parfaite, parce que surpuissante, vis-à-vis de l'imperfection et de la misère humaines. Le démon est ainsi la seule possibilité d'accès à la nature: c'est par son inspiration que la perfection de celle-ci apparaît au poète. Nous nous trouvons donc face à un triangle dont les côté sont l'homme, la nature et le démoniaque; le démoniaque assure le lien entre l'homme et la nature dans ce mouvement contradictoire de perte et de possession.

C'est dans la troisième strophe de la seconde rédaction que nous voyons prendre corps le protagoniste de L'Ange de la Mort. L'une des activités de cet esprit consiste à persécuter les mourants: lorsque l'âme, apeurée, descend dans la tombe, il l'accompagne, mais pas pour la réconforter. Ce que l'esprit fait au lieu d'apporter du réconfort au mourant, nous le découvrons dans L'Ange de la Mort. 


\section{L'Ange de la Mort}

Lermontov compose L'Ange de la Mort dans la même année $1831 .^{9}$ Le poème, de 550 vers, raconte l'histoire d'une chute. L'histoire se déroule en Hindoustan: au début de l'histoire, l'ange de la mort apporte du réconfort aux mourants, pour que leurs derniers instants soient doux. Ému par le désespoir que la mort de la jeune Ada a causé à son compagnon, Zoraïm, il ranime son corps et prend sa place à côté de Zoraïm. Mais celui-ci, attiré par la gloire, part à la guerre et est tué sur le champ de bataille. Déçu par les hommes, l'ange devient une créature méchante: c'est désormais pour les tourmenter qu'il approche les mourants. C'est donc l'esprit perfide de Mon Démon dont la genèse est ici racontée.

Un passage semble important pour identifier la relation de Lermontov au démon. Il s'agit aussi de l'un des passages les plus réussis du poème. Après que Zoraïm a annoncé à Ada sa décision de partir à la guerre, Ada lui répond par ces vers (293307):

«N'as-tu pas vu comment le ruisseau

Reflète la fleur qui s'y penche?

Quand l'eau ne remue pas,

La fleur, immobile, en elle se contemple,

Mais si la brise fraîche

Vient troubler la verte vague,

Et que s'agite la vague,

L'ombre de la petite fleur peut-elle

Ne pas flotter, comme flotte la vague?

Aussi irrévocablement le sort

A lié mon destin à ton destin:

Cette vague, c'est toi, et moi je suis cette fleur.

Tu es triste, je suis triste avec toi.

Qui peut le savoir? ce moment, peut-être,

Est le dernier de notre bonheur! ....110

9 À ma connaissance, ce poème n'a jamais été traduit en français. La traduction que j'en ai faite, et que je citerai ici, sera publiée dans la revue Po\&sie 175 (2021), p. 115-131.

10 Sotch., t. III, p. 140-141. 
Ici l'Ange possédant le corps d'Ada (ангел-дева, vient de l'appeler Lermontov, v. 292) s'identifie à la fleur, et elle identifie Zoraïm au reflet dans l'eau («Cette vague, c'est toi, et moi je suis cette fleur»: plus littéralement, «La vague c'est ton image, la mienne c'est la fleur», «Волна - твой образ, мой - цветок»). L'objet reflété est actif par rapport à son reflet: l'emprise vient alors de l'élément surnaturel (de l'ange qui bientôt deviendra un démon). Et Zoraïm subit cette emprise.

Les derniers vers du poème sont particulièrement révélateurs: l'être surnaturel abandonne le corps d'Ada pour devenir l'être perfide que décrit la seconde rédaction de Mon Démon; Zoraïm, l'homme, est mort d'une mort stupide; la nature se montre alors dans toute sa majestueuse beauté (v. 480-487):

Entretemps au sommet de la voûte

Une étoile adamantine

Apparut dans sa splendeur immuable,

Pure, belle comme toujours,

Et son rayon semblait ignorer

Ce qu'il éclairait sur la terre:

Ainsi, enjoué, il descendait

Sur la proie des vers et des tombeaux. ${ }^{11}$

C'est donc une nature gaie (v. 486: «игриво») qui exhibe, muette et splendide, sa perfection devant les hommes et les démons. Et encore une fois, Lermontov nous confronte à cet inéluctable triangle - la nature, l'homme, le démoniaque scellant le drame de l'existence.

\section{Le Démon}

Dans Le Démon, Lermontov a sûrement atteint l'un des sommets de son art poétique. Cela ne va pas sans un affinement de sa conception du démoniaque, dont désormais les caractères théoriques sont développés avec maîtrise et lucidité. Le démon - à la fois protagoniste du poème et inspirateur de Lermontov - est désormais l'incarnation de la figure irrationnelle

11 Ibidem, p. 146 
par excellence, c'est-à-dire la contradiction. Et il l'est tant dans ses propos que dans son incarnation poétique: le démon n'est pas seulement celui qui trouble le monde humain et le monde de la nature par des concepts impossibles qui expriment la nécessité de son essence, il est aussi un être psychologiquement et formellement ambigu.

Proscrit du ciel, le démon tombe amoureux d'une mortelle, la belle Tamara, qu'il aperçoit survolant le château de son père, en Géorgie. Le démon réussira à faire tuer le fiancé que Tamara attend, puis, lorsqu'elle se réfugie dans un monastère, il la poursuit. Au centre du poème, se trouve la rencontre nocturne du démon et de Tamara: le démon la tuera en effleurant ses lèvres avec sa bouche. Puis, lorsqu'il la conduira dans son royaume, un ange du ciel lui enlèvera sa proie, qui sera portée au ciel.

C'est aux yeux de Tamara, et bien avant la fatale rencontre nocturne avec le démon, que le trait fondamental de la psychologie du démon apparaît. Poursuivie par sa voix, par sa présence, alors qu'elle est déjà entrée au monastère, Tamara sent qu'elle n'a affaire ni à son ange gardien, ni à un esprit infernal, mais que l'esprit (390-391):

... ressemblait à un soir clair:

$\mathrm{Ni}$ jour, ni nuit, - ni ténèbres, ni lumière! ${ }^{12}$

Le démon est perçu par Tamara comme une créature de la limite, limite entre deux réalités opposées comme le sont le jour et la nuit. Notons que cet être liminaire colore tout le poème et investit pleinement la nature telle qu'elle y est représentée. En effet, la couleur du poème est une couleur bleuâtre, c'est-àdire la couleur de la nuit qui, timidement, prend les couleurs de jour, ou bien du jour qui s'obscurcit graduellement. Ainsi, le crépuscule et l'aurore sont des moments poétiques clefs du poème.

D'autres éléments de la nature lermontovienne évoquent ce statut: ainsi la rosée, souvent évoquée ${ }^{13}$, ce reste de la nuit et de son humidité qui se manifeste au lever du jour. On observera

12 Sotch., t. IV, p. 195.

13 V. $80,138,314,354,859-860$. 
également la présence constante de nuages, brumes, vapeurs et fumées, des éléments qui à la fois cachent et rendent visible la lumière, le mouvement et la nature; et encore le brouillard, qui apparaît sûrement comme l'un des principaux éléments du démon. Ainsi, parlant de son désespoir, le démon se décrit «De foudre et de brouillard vêtu» (v. 719), vers qui évoque la description de Mon Démon; Tamara perçoit la présence du démon comme celle d'un intrus «brumeux» (туманный: v. 378). Се n'est certainement pas un hasard si la première action du démon dans le déroulement du poème coïncide avec le lever du brouillard: le fiancé de Tamara, Sinodal, est attaqué par les Ossètes, suite à une ruse du démon, précisément lorsque surgit le brouillard à la lisière entre la nuit et le jour (213-215):

Il est déjà tard. Sur le sommet enneigé

La rougeur s'évanouit ; le brouillard se leva ...

La caravane accéléra. ${ }^{14}$

Il semble aussi que Lermontov ait voulu instituer des analogies entre les acteurs 'personnels' du poème et les acteurs 'naturels', justement pour renforcer le caractère 'liminaire' de sa vision. Les différents plans de la réalité font écho l'un à l'autre. Notons ainsi que la danse de Tamara sur le toit du château de Goudal - formellement, l'un des plus hauts passages du poème - évoque la course frénétique du cheval qui amènera le corps sans vie du fiancé. Voici Tamara et ses compagnes (v. 112-126):

Battant des mains en cadence

Elles chantent - et de son tambourin

S'empare la jeune fiancée.

Et voici qu'avec une main

Le faisant voltiger au-dessus de sa tête,

Tantôt elle vole plus légère que l'oiseau,

Tantôt s'arrête, regarde -

Et son regard humide brille

Par dessous ses cils envieux;

Tantôt elle hausse le noir sourcil,

Tantôt elle se penche légèrement,

Et sur le tapis glisse, vogue

Son petit pied divin;

Et elle sourit,

Pleine d'une gaîté d'enfant. ${ }^{15}$

14 Sotch., t. IV, p. 190.

15 Ibidem , t. IV, p. 186-187. 
C'est par le même mouvement, alternant arrêts et accélérations, que le cheval amène le cadavre de son maître au festin nuptial (v. 268-277):

Il fuit, le cheval plus rapide que la biche,

Il s'ébroue et s'épuise, comme s'il allait à la bataille;

Tantôt il arrête sa course,

Tend l'oreille à la brise,

En gonflant ses naseaux;

Tantôt, en frappant

La terre de ses sabots ferrés,

Et secouant sa crinière ébouriffée,

Il vole en avant frénétiquement,

Monté par un cavalier silencieux!16

De même, lorsque le démon promet à Tamara qu'il viendra la visiter au lever de la nuit, il évoque entre autres l'image splendide du vol nocturne d'un petit oiseau (v. 345-359):

«Dès que la nuit de son voile

Recouvrira les cimes du Caucase ;

Dès que le monde, d'une parole magique

Ensorcelé, se taira ;

Dès que le vent sur le rocher

Agitera l'herbe fanée,

Et que l'oiselet, qui s'y cache,

Voltigera gaîment dans les ténèbres ;

Et que sous le pied de vigne,

Buvant goulûment la rosée céleste,

Éclora la fleur de la nuit ;

Dès que la lune dorée

Doucement se lèvera de la montagne

Et furtivement te regardera,

Alors vers toi je volerai .... $\rangle^{17}$

Le mouvement de l'oiselet n'est pas sans rappeler le mouvement de l'ange vainqueur qui, à la fin du poème, soustrait définitivement au démon l'âme de Tamara (v. 1051-1054):

Et l'ange avec des yeux sévères

Regarda le tentateur

Puis, d'un joyeux battement d'ailes,

Se recula dans la lumière du ciel. ${ }^{18}$

16 Ibidem, p. 191-192.

17 Sotch., t. IV, p. 194.

18 Ibidem, p. 216. 
Dans ce même passage, la fleur de la nuit qui boit la rosée reprend, sous une lumière gracieuse, l'image sinistre de la seconde rédaction de Mon Démon, décrivant le démon qui avale la fumée de la bataille et les vapeurs sanguines, deux lieux où Lermontov a recours aux mêmes termes: «Глотает жадно дым сраженья/ И пар от крови пролитой» (Mon Démon, seconde rédaction, v. 15-16), «Росу небес глотая жадно,/ Цветок распустится ночной» (Le Démon, v. 354-355).

Malgré leur séparation radicale, les mondes humain, démoniaque et naturel renvoient donc constamment l'un à l'autre. Le démoniaque est certes un élément de fiction, mais il est, peut-être plus fondamentalement encore, la forme par laquelle Lermontov prend conscience de sa séparation d'avec la nature.

Dans Le Démon, le triomphe de la contradiction se produit dans le serment. En effet, tout le serment est construit sur un enchaînement de contradictions, particulièrement manifeste au début. Le démon est un être intrinsèquement illogique; et il utilise comme instrument de séduction la manifestation effrontée de ces contradictions, cela dans quelques cent vers, unanimement reconnus comme l'un des plus hauts moments du poème. Tamara, qui a désormais compris la nature diabolique de son séducteur, vient de demander au démon d'abdiquer son «méchant empire» (v. 769, злое стяжание), ${ }^{19}$ demande à laquelle le démon répond ainsi (v. 773-794):

«Je le jure par le premier jour de la création,

Je le jure par son dernier jour,

Je le jure par notre rencontre

Et par la séparation qui à nouveau nous menace.

...

Je le jure par le bonheur et le malheur,

19 H. Grégoire traduit «0т злых стяжаний» par «les séductions pécheresses» (타, p. 311); en réalité стяжание est un terme de la langue scripturaire signifiant la richesse, les biens, l'abondance: voir Job, 20, 28: «Исчезнет стяжание дома его»; Proverbes, 10, 3: «Не допустит Господь терпеть голод душе праведного, стяжание же нечестивых исторгнет»; ou encore le composé любостяжание, c'est-à-dire 'amour des richesses', ou 'avarice' (Ép. aux Éphésiens, 5, 3; Ép. aux Colossiens, 3, 5). 
J'ai renié l'ancienne vengeance,

J'ai renié les pensées orgueilleuses .... ${ }^{20}$

Ici, le démon est clairement en train de polariser l'attention de Tamara sur des couples d'antonymes, sans que cela puisse être compris comme une ruse: la ruse consisterait à cacher sa nature illogique, or le démon a recours à la stratégie contraire, puisqu'il exhibe cette nature. Dans la partie du serment qui suit, il se présente, à quelques vers de distance, comme un repenti voulant expier ses péchés et comme le prince du mal, les deux qualifications devant servir à attirer Tamara dans ses bras (v. 797-802, 845-852):

«Avec le ciel je veux me réconcilier,

Je veux aimer, je veux prier,

Je veux croire au bien.

Par la larme du repentir j'effacerai

De mon visage, digne de toi,

Les traces du feu céleste

Oh non, sublime créature,

Un autre destin t'est réservé,

Une autre souffrance t'attend,

La profondeur d'autres ivresses.

Laisse les anciens désirs

Et ce pauvre monde à son destin:

L'abîme de la connaissance orgueilleuse,

Je te l'ouvrirai en échange.» ${ }^{21}$

Les deux promesses sont manifestement incompatibles: de la réconciliation avec le ciel à la connaissance orgueilleuse (v. 801, гордое познание), c'est par des propos incohérents que le démon anéantit Tamara.

Le serment du démon s'achève, terrible, sur un impératif: «Aime-moi!» (v. 872: «Люби меня!»). ${ }^{22}$ Le démon effleure de ses lèvres celles de de Tamara, et ce baiser empoisonné tue Tamara. Lermontov décrit ici le démon se tenant au-dessus de Tamara, couchée, droit comme un poignard (v. 878-880):

20 Sotch., t. IV, p. 208.

21 Ibidem, p. 208, 210.

22 Ibidem, p. 210. 
Il la brûlait. Dans les ténèbres de la nuit

Il étincelait, et se tenait droit au-dessus d'elle, Irrésistible, comme un poignard. ${ }^{23}$

L'identification du démon au poignard est intéressante, puisque le poignard (кинжал) est l'une des figures auxquelles Lermontov s'est lui-même identifié, dans deux poèmes de la même année 1838: Le Poète, et Le Poignard. Il écrit, dans ce dernier (v. 13-16):

Comme un compagnon tu m'es donné, gage muet d'amour, Ton exemple n'est, pour le pèlerin, pas vain:

Oui, je ne changerai pas, et mon âme sera forte,

Comme toi, comme toi, mon ami de fer. ${ }^{24}$

On notera encore que d'une part le démon promet à Tamara de l'enlever au monde, de la soulever au sommet du ciel, où elle observera le monde de loin et dédaignera ce lieu privé de bonheur (v. 813-824);25 de l'autre, il lui promet de lui faire don du monde avec toutes ses splendeurs (v. 853-871: «Я дам тебе всё, всё земное»).

Une fois encore, c'est en lui promettant des souffrances que le démon essaie de séduire Tamara (v. 845-848):

«Oh non, sublime créature,

Un autre destin t'est réservé,

Une autre souffrance t'attend,

La profondeur d'autres ivresses.» ${ }^{26}$

Et à Tamara, qui essaie de résister au séducteur en évoquant Dieu puis les peines de l'enfer, il réserve la plus terrible des réponses (v. 745-749):

Tamara

«On pourrait nous entendre! ...»

Le démon

«Nous sommes seuls.»

23 Ibidem, p. 211.

24 Sotch., t. II, p. 108. Cf. EP, p. 94.

25 Nous ignorons si et comment Lermontov en ait pris connaissance, mais la vision cosmique est un topos de la littérature théologique médiévale. Dans la vision de saint Benoit, dans la Consolation de Boèce et dans la Commedia de Dante, où, comme chez Lermontov, la vision permet de voir la misère du monde: voir Paradiso, xxii (v. 151-153): «L'aiuola che ci fa tanto feroci,/volgendom'io con li etterni Gemelli,/tutta m'apparve da' colli a le foci.»

26 Sotch., t. IV, p. 210. 
Tamara

«Et Dieu donc!»

Le démon

«Sur nous il ne jettera pas son regard:

Il se soucie du ciel, pas de la terre!»

Tamara

«Et le châtiment, les supplices de l'Enfer?»

Le démon

«Et alors? Tu y seras avec moi!!» ${ }^{27}$

"Tu y seras avec moi», comme pour dire que les supplices éternels devraient être supportables, et même souhaitables pour Tamara, à partir du moment où elle les partagera avec son amant. Ce n'est pas un simple artifice littéraire: dans un poème plus intime, «Écoute, peut-être, ...» (1832), Lermontov rêve d'une jonction contradictoire entre un moi-démon et une femme-ange:

Écoute, peut-être, quand nous quitterons

Ce monde à jamais, où nos âmes ont si froid,

Peut-être au pays où inconnue est la fraude,

Tu seras un ange, et moi un démon!

Jure alors d'oublier, ma chère,

Pour ton ancien ami, tout le bonheur du ciel!

Que le triste proscrit, par le destin condamné,

Soit pour toi le paradis, et toi pour moi, l'univers! ${ }^{28}$

L'exigence de Lermontov et celle du démon est la même: que la femme aimée renonce au paradis, pour trouver son bonheur aux enfers.

\section{Conclusions}

De quoi l'obsession démoniaque lermontovienne est-elle la figure? Il faut probablement distinguer différents niveaux: en tant qu'il entretient des relations avec le monde humain, le démon est une muse, un destin, ainsi que l'un des moteurs des actions de la vie. Comme 'sceau théorique', la fonction du

27 Ibidem, p. 206-207.

28 Sotch., t. II, p. 40. 
démon est simple: antagoniste de Dieu, de la nature et des hommes, le démon représente une sorte d'anti-fondement. $\mathrm{Ni}$ Dieu, ni la nature, ni les hommes ne peuvent tolérer la contradiction: tel est le pas qui ne pourra jamais être franchi pour réintégrer le démon à l'un de ces mondes. Or si le principe de non-contradiction permet de penser et de parler, son renversement est la forme la plus pure de révolte contre le monde. La rébellion de Lermontov est à sa racine logique.

Cela explique que dans l'un de ses poèmes les plus célèbres, «Je m'en vais tout seul sur la grand' route» («Выхожу один я на дорогу ...», 1841), considéré presque comme un testament spirituel, Lermontov exprime le désir paradoxal d'être simultanément vivant et mort, cette coexistence de vie et de mort prenant la forme d'un sommeil éternel (vv. 9-16):

De la vie, je n'attends déjà plus rien, Je ne regrette rien du passé; Je cherche la liberté, la paix! Je voudrais m'oublier, m'assoupir!

Mais non pas du froid sommeil de la tombe ... Je voudrais à jamais m'endormir ainsi, Qu'en ma poitrine somnolent les forces de la vie, Que la poitrine, respirant, se soulève doucement. ${ }^{29}$

Une variation sur le même thème se trouve dans Rêve (1841):

Sous la chaleur du midi dans une vallée du Daghestan Inerte je gisais, du plomb dans la poitrine; Encore fumait la blessure profonde, Et goutte à goutte coulait mon sang.

Je gisais seul sur le sable de la vallée; Autour s'amassaient les escarpements rocheux, Le soleil brûlait leurs cimes jaunes

Et il me brûlait - mais je dormais d'un sommeil de mort.

Et je rêvais, resplendissante de lumières, Une soirée de fête dans mon pays natal.

Parmi les jeunes femmes, couronnées de fleurs, À mon sujet on conversait gaiement. Mais sans prendre part à la gaie conversation, Une femme était assise là-bas, l'air songeur, 
Et sa jeune âme dans un triste rêve,

Dieu sait pourquoi, était plongée;

Elle rêvait d'une vallée du Daghestan;

Un cadavre connu gisait dans cette vallée;

Dans sa poitrine fumait la noire blessure,

Et le sang se répandait en un froid ruissellement. ${ }^{30}$

Le mouvement du poème ${ }^{31}$ est ainsi celui d'un rêve infini, donc d'un sommeil éternel: le premier rêve (la vision du poète) a pour objet un deuxième rêve (le rêve du mourant) qui a pour objet un troisième rêve (le rêve de la femme), qui a pour objet le premier rêve, ce qui fera recommencer le cycle onirique, si bien que ce mouvement de rêves qui s'emboîtent est en réalité une régression à l'infini. Il s'agit également d'une vision contradictoire, dans la mesure où chacun des rêves est simultanément cause et effet des autres.

Nous nous sommes proposés d'éclaircir la nature du démonisme de Lermontov; notre brève enquête nous a amené à associer ce démonisme à la contradiction. Or la contradiction lermontovienne n'est pas, comme chez Hegel, le moteur de l'esprit, puisque la contradiction lermontovienne ne donne lieu à aucune synthèse; ${ }^{32}$ ce n'est pas non plus l'expression d'un scepticisme primaire, ni un simple artifice de style. La contradiction démoniaque a ceci de particulier, qu'au lieu de se nier et par elle-même pour permettre son propre dépassement, elle demeure indestructible, maintenant le poète dans une aporie sans fin. ${ }^{33}$

30 Ibidem, p. 197. Cf. EF, p. 61-62. Les deux poèmes, Rêve et «Je m'en vais tout seul sur la grand' route», sont de 1841, année de la mort de Lermontov. II est difficile de ne pas y lire un présage. V. Nabokov a livré une analyse brillante du poème, en en rapprochant la structure à celle du roman Un héros de notre temps: LERMONTOV, 2002, pp. 6-15.

31 Selon NABOKOV, ibid., p. 7, «a spiral bringing us back to the first stanza.»

32 Voir REID, 1982, pp. 189-210: p. 194: «Udodov sees these antinomies as theses and antitheses of a modified Hegelian dialectic which finds its most explicit statement in the Demon's klyanus' speech», c'est-à-dire dans le serment; et ibid., note 28: "The modification, according to this critic, is the effective absence of synthesis.»

33 Je remercie, pour leurs conseils, encouragements et relectures, Guillaume Navaud et Philippe Büttgen. 


\section{Références bibliographiques}

GREGORY, T. Principe di questo mondo. Il diavolo in Occidente, Roma-Bari, Editori Laterza («i Robinson / Letture»), 2013.

GRIBOÏĖDOV, A., LERMONTOV, M. POUCHKINE, A. Euvres, éd. G. Aucouturier et al., Paris, Éditions Gallimard («Bibl. de la Pléiade»), 1973.

KROPOTKIN, P. Russian Literature, New York, McClure, Phillips \& Co., 1905.

LERMONTOV, M. Y. Sotchinéniya $v$ shésti tomakh, éd. N. F. Bél'tchikov, B. P. Gorodétskij, B. V. Tomashévskij, Moscou-Leningrad, Ak. Naoukh SSSR, 1954-1957.

LERMONTOV, M. Y. EEuvres Poétiques, publiées sous la direction d'E. Etkind, Lausanne[-Paris], L'Âge d'Homme («Classiques Slaves»), 1985.

LERMONTOV, M. Y. A Hero of Our Time, translated by V. Nabokov in collaboration with D. Nabokov, Woodstock \& New York, Ardis Publishers, 2002.

LERMONTOV, M. Y. Un héros de notre temps. Герой нашего времени, éd. D. Lévy-Bertherat, Paris, Éditions Flammarion, 2003.

REID, R. «Lermontov's Demon: A Question of Identity», The Slavonic and East European Review, 60/2 (Avril 1982).

Recebido em: 31/03/2021

Aceito em: 11/04/2021

Publicado em abril de 2021 